\title{
Dynamics of Nutrient-Phytoplankton Interaction in the Presence of Viral Infection and Periodic Nutrient Input
}

\author{
K. pada Das, S. Chatterjee, J. Chattopadhyay ${ }^{1}$ \\ Agricultural and Ecological Research Unit, Indian Statistical Institute, \\ 203 B. T. Road Kolkata 700108, India
}

\begin{abstract}
Chattopadhyay et al. [Biosystems (2003), 68, pp. 5-17] proposed and analyzed an $N-P$ model in the presence of viral infection on phytoplankton population. They studied the dynamics under the constant nutrient input. The present paper deals with the problem with seasonal variability on nutrient input. We use a general periodic function for nutrient input. We observe the dynamics of the system by considering (i) the infected phytoplankton consumes nutrient and (ii) the infected phytoplankton is not in a state to consume nutrient. Conditions for the persistence and extinction of populations are worked out. Our numerical experiments show that if the infected phytoplankton does not take nutrient then susceptible phytoplankton coexists with the infected ones. But if the infected phytoplankton consumes nutrient then there is a chance for extinction of susceptible phytoplankton for high rate of infection. We also observe that periodic nutrient input enforces the system to enter into chaotic region.
\end{abstract}

Keywords: nutrient-phytoplankton, periodic nutrient recycle, viral disease, chaos AMS subject classification: 92B05, 92D25.

\section{Introduction}

Phytoplanktons are the micro organism in aquatic system. They transform mineral nutrients into primitive biotic material using external energy, provided by the sun. The dynamical relationship between the phytoplankton community and the nutrients has long been of great interest from both experimental and theoretical view point. The importance of nutrients on the growth of plankton leads to explicit incorporation of nutrients concentration in the plankton- herbivore models. Busenberg et al. [29] analyzed the dynamics of plankton-nutrient interaction and observed that under certain conditions the coexistence of phytoplankton and zooplankton occured in an orbitally stable

\footnotetext{
${ }^{1}$ Corresponding author. E-mail: joydev@isical.ac.in
} 
oscillatory mode. Ruan [31] considered plankton-nutrient interaction model with general nutrient uptake function and instantaneous nutrient recycling. Further he considered the plankton-nutrient interaction model with a fluctuating nutrient input and with a periodic washout rate. He showed that coexistence of the zooplankton and the phytoplankton might arise due to positive bifurcating periodic solutions. Many research papers related to nutrient-plankton model with constant and periodic nutrient input have been published, for example see [12, 4, 3, 18]. In recent time, Huppert et al.[2] considered a nutrient-phytoplankton model, where they assumed the consumption rate to be a function of time $t$. But they took the input rate of the nutrient to be a constant. They observed the seasonal phytoplankton bloom and the dynamics of seasonally recurring algae blooms. Their model displayed a wide spectrum of dynamical behaviours, from simple cyclical blooms which trigger annually, to irregular chaotic blooms in which both the time between outbreaks and their magnitudes were erratic. Viral infection is another factor, other than the nutrient availability, that govern the growth rate of the phytoplankton population. In marine ecosystem phytoplankton are infected through virus entities that are the most abundant in the sea-nearshore and offshore, tropical to polar, sea surface to seafloor, and in sea ice and sediment pore water. The first evidence of infective "phycoviruses" in sea water was provided by Mayer and Taylor [17]. Quite a good number of studies $[22,27,21,6]$ showed the presence of pathogenic viruses in the phytoplankton communities. Fuhrman [16] synthesized the accumulated evidence regarding the nature of marine viruses and their ecological as well as biogeological effects. Suttle et al. [6] showed by using electron microscopy that the viral disease could infect bacteria and even phytoplankton in coastal water. Viral infections can cause various phenomenon such as phytoplankton cell lysis, the collapse of Emiliania huxleyi blooms in mesocosms [11] and in the North Sea [5] and the lysis of Chrysochromulina [7]. Due to strain-specific, viruses can increase genetic diversity [20]. Viruses may be important agents in the mortality of marine micro-organism and in controlling their genetic compositions. Nevertheless, despite the increasing number of reports, the role viral infection in phytoplankton population is still far from understood.

Recently, Chattopadhyay et al. [15] proposed a nutrient-phytoplankton model with viral infection on phytoplankton population. They divided phytoplankton into two groups susceptible and infected. They derived the conditions for the co-existence and extinction of the population. Their results indicated that the system will persist when the infected phytoplankton population was not able to consume nutrient.

The main aim of the present paper is to observe the effect of viral disease on the growth of phytoplankton in the presence of periodic nutrient input. As far our knowledge goes, no such work has been initiated in this direction. In this paper we modify the model of Chattopadhyay et al. [15] by introducing periodic nutrient input. We first consider a phytoplankton-nutrient interaction model with general uptake functions and input nutrient is general periodic function of period $\omega$. Later for numerical simplicity we consider the functional response is of Holling-II. Then we compare our system numerically with the dynamics of the system where infected phytoplankton is incapable of nutrient uptake and the system where input nutrient is of constant in nature.

The paper is organized as follows. In section 2, we outline the basic mathematical model with some preliminary results. In section 3 , we analyze our model with the assumption that the infected phytoplankton population is incapable of nutrient consumption. We perform extensive numerical 
simulations in section 4 . The article ends with a concluding remarks.

\section{Model description and qualitative analysis}

\subsection{The mathematical model}

We modify the model of Chattopadhyay et al. [15] which studied the nutrient plankton interaction under the influence of viral infection, by introducing periodic nutrient input $N^{0}(t)$, a nontrivial positive $\omega$ periodic $C^{0}$ function. Thus the mathematical model takes the following form,

$$
\begin{aligned}
\frac{d N}{d t} & =D\left(N^{0}(t)-N\right)-a S f_{0}(N)-b I f_{1}(N) \\
\frac{d S}{d t} & =a S f_{0}(N)-\lambda \frac{S I}{S+I}-D_{1} S \\
\frac{d I}{d t} & =b I f_{1}(N)+\lambda \frac{S I}{S+I}-D_{2} I
\end{aligned}
$$

System (2.1) has to be analyzed with the following initial conditions :

$$
N(0)=N_{0}>0, S(0)>0, I(0)>0
$$

Here, $N(t)$ is the concentration of the nutrient at time $t . S(t)$ and $I(t)$ be the concentration of susceptible phytoplankton population and infected phytoplankton respectively at time t. $N^{0}(t)$ be the periodic input of nutrient concentration; $D$ is the washout rate of the nutrient; $D_{1}$ is the mortality rate of susceptible population; $D_{2}$ is the mortality rate of infected population and $\lambda$ denotes the infection rate. The maximal nutrient uptake rate for the susceptible population and maximal nutrient uptake rate for the infected population are $a$ and $b$ respectively.

A crucial part of all host-pathogen models is the term describing the way in which the pathogen is transmitted between hosts. In this connection we mention that all conventional host-pathogen models make the assumption that the rate of pathogen transmission follows the mass action law $\eta S I$ [i.e. the product of the number of susceptible hosts (S), the number of infectious units (I) and the transmission coefficient, a constant which represents the chance of transmission of the infectious per contact between susceptible hosts and infectious units] [28, 25]. But Jong et al. [8] and Bouma et al. [1] pointed out that there was some confusion between mass action incidence $\eta S I$ and standard incidence $\frac{\lambda S I}{S+I}$ (where $S+I$ is total host population) on the description of disease transmission. They also suggested that standard incidence $\frac{\lambda S I}{S+I}$ is more suitable term to describe the disease transmission. Begon and his co-workers $[23,24]$ concluded that standard incidence is better descriptor of transmission dynamics than density dependent transmission for cowpox. In our proposed model standard incidence has been assumed to transmit viral disease in phytoplankton populations rather than a simple mass action $\eta S I$. The comparison between standard incidence and mass action incidence shows $\lambda=\eta(S+I)$ so that this form implicitly assumes that the contact rate $\lambda$ increases linearly with the population size [14]. But for human disease the contact rate seems to be only very weakly dependent on the population size [14]. Also there are animal 
population like mice in a mouse-room or animal in a herb [8], where the disease transmission primarily occurs locally from nearby animals. This is because in case of large population and slow movements of individual prevents it to make contact to a large numbers of individuals in a unit time like phytoplankton. In this connection we mention that Beltrami and Carroll [9] assumed standard incidence in phytoplanktons so that infected phytoplanktons represent a small fraction of the total biomass. In fact, this assumed that the contact rate was not limited by the availability of phytoplankton cells, which was responsible in view of the typically high algal concentrations. The above theoretical and experimental evidences motivate us to consider the transmission term as of standard incidence.

Here the functions $f_{0}(N)$ and $f_{1}(N)$ describe the nutrient uptake rates of susceptible and infected population respectively. Moreover we assume

(A1) $N^{0}(t)$ is a nontrivial positive $\omega$-periodic $C^{0}$ function.

(A2) $f_{i}: R_{+} \rightarrow[01], i=0,1, f_{i} \in C^{1}\left(R_{+},\left[\begin{array}{ll}0 & 1\end{array}\right]\right)$, is increasing i.e. $\left(f_{i}(r)>0\right.$ for $r \geq 0, i=$ $0,1)$ and $f_{i}(0)=0, f_{i}(+\infty)=1$.

Suppose

$$
l_{1}=\min \left\{N^{0}(t): 0 \leq t \leq \omega\right\}, l_{2}=\max \left\{N^{0}(t): 0 \leq t \leq \omega\right\}
$$

and

$$
\hat{N}=\frac{1}{\omega} \int_{0}^{\omega} N^{0}(t) d t
$$

Then clearly $0<l_{1}<\hat{N}<l_{2}$.

$$
\dot{N}=D\left(N^{0}(t)-N\right)
$$

It is easy to verify that

$$
N^{*}(t)=\frac{D}{e^{D \omega}-1} \int_{0}^{\omega} e^{D r} N^{0}(t+r) d r
$$

is the unique $\omega$-periodic solution of (2.2) and moreover every solution of (2.2) can be written as $N(t)=N^{*}(t)+c e^{-D t}$ with $c=N(0)-N^{*}(0)$.

We also found that $N(t)-N^{*}(t) \rightarrow 0$ as $t \rightarrow \infty$ and thus $N^{*}(t)$ is a globally exponentially stable $\omega$-periodic solution of (2.2).

Since $l_{1} \leq N^{0}(t) \leq l_{2}$, all solutions $\mathrm{N}(\mathrm{t})$ eventually enter the interval $J=\left[l_{1} l_{2}\right]$ and remain there for all future time.

\subsection{Some preliminary results}

The following lemma describes the behaviour of $N^{*}(t)$.

Lemma 1. (i) There exists $b>0$ such that

$$
l_{1}+b \leq N^{*}(t) \leq l_{2}-b
$$


for all $t \in R$.

(ii)

$$
\frac{1}{\omega} \int_{0}^{\omega} N^{*}(t) d t=\hat{N}
$$

\section{Proof.}

Since all solutions $N(t)$ eventually enter the interval $J=\left[l_{1} l_{2}\right]$ and remain there for all future time, $l_{1} \leq N^{*}(t) \leq l_{2}$.

Now if we take

$$
l_{2}=\max \left\{N^{*}(t): 0 \leq t \leq \omega\right\}\left(\text { or } l_{1}=\min \left\{N^{*}(t): 0 \leq t \leq \omega\right\}\right)
$$

then there exists $t^{*} \in(0, \omega]$ such that $N^{*}\left(t^{*}\right)=l_{2}\left(\right.$ or $\left.=l_{1}\right)$. Now (2.2) has no constant solution since $N^{0}(t)$ is not constant, hence there exists $t_{0} \in\left(0, t^{*}\right)$ such that $N^{*}\left(t_{0}\right)<l_{2}\left(\right.$ or $\left.>l_{1}\right)$. Hence we can choose a solution $\mathrm{N}(\mathrm{t})$ with $N^{*}\left(t_{0}\right)<N\left(t_{0}\right)<l_{2}$ ( or $l_{1}<N\left(t_{0}\right)<N^{*}\left(t_{0}\right)$ ). Then by the intermediate value theorem, there exists $t_{1} \in\left(t_{0}, t^{*}\right]$ such that $N\left(t_{1}\right)=N^{*}\left(t_{1}\right)$ since $N\left(t_{0}\right)>N^{*}\left(t_{0}\right)$ (or $N\left(t_{0}\right)<N^{*}\left(t_{0}\right)$ ) while $N\left(t^{*}\right) \leq N^{*}\left(t^{*}\right)$ (or $N\left(t^{*}\right) \geq N^{*}\left(t^{*}\right)$ ). This is a contradiction to the uniqueness of solutions of initial value problems. So there exists $b>0$ such that

$$
l_{1}+b \leq N^{*}(t) \leq l_{2}-b \text { for all } t \in R .
$$

Now, we proof the second part of the theorem.

(ii) By substituting $N^{*}(t)$ into (2.2) and then integrating both side of (2.2) from 0 to $\omega$ we get

$$
\frac{1}{\omega} \int_{0}^{\omega} N^{*} d t=\frac{1}{\omega} \int_{0}^{\omega} N^{0}(t) d t=\hat{N} .
$$

Lemma 2. Suppose (A2) holds. Then each solution $(N(t), S(t), I(t))$ of $(1)$ with its initial value in $R_{+}^{3}$ will remain in $R_{+}^{3}$ for all $t \geq 0$. Further if we denote $u(t)=N^{*}(t)-N(t)$ for solutions $N(t)$, then the following are true:

(i)

$$
u(0) e^{-D t} \leq u(t),
$$

(ii) Either there exists $t_{1} \geq 0$ such that $u(t) \geq 0$ for all $t \geq t_{1}$ or $u(t)<0 \forall t \geq 0$ in which case $u(t), S(t), I(t)$ all tend to zero exponentially as $t \rightarrow+\infty$.

Proof.

$$
\begin{aligned}
& u(t)=N^{*}(t)-N(t) . \\
\dot{u(t)}= & N^{*}(t)-\dot{N(t)} \\
= & D\left(N^{0}(t)-N^{*}(t)\right)-D\left(N^{0}(t)-N(t)\right)+a S f_{0}(N)+b I f_{1}(N) \\
= & -D u(t)+a S f_{0}(N)+b I f_{1}(N) \\
\geq & -D u(t)
\end{aligned}
$$

By standard comparison theory it follows that

$$
u(0) e^{-D t} \leq u(t) .
$$


On the other hand, by variation of constant

$$
\begin{gathered}
u(t)=e^{-D t}(u(0)+F(t)) \\
\dot{u(t)}=-D e^{-D t}(u(0)+F(t))+e^{-D t} \dot{F(t)} .
\end{gathered}
$$

From (2.3) and (2.5) we have

$$
-D e^{-D t}(u(0)+F(t))+e^{-D t} \dot{F(t)}=-D u(t)+a S f_{0}(N)+b I f_{1}(N) .
$$

Thus from (2.4) we have

$$
-D e^{-D t}(u(0)+F(t))+e^{-D t} \dot{F(t)}=-D e^{-D t}(u(0)+F(t))+a S f_{0}(N)+b I f_{1}(N) .
$$

Therefore

$$
F(t)=\int_{0}^{t} e^{D r}\left[a S(r) f_{0}(N)+b I(r) f_{1}(N)\right] d r,
$$

is an increasing nonnegative function for $t \geq 0$ since the integrand is positive.

Therefore, the first case of (ii) occurs if $u(0) \geq 0$. If $u(0)<0$ then $F(+\infty)>-u(0)$, otherwise $u(t)<0 \forall t \geq 0$.

In the latter case, let

$$
\begin{aligned}
& W(t)=S(t)+I(t)-u(t), \\
\dot{W(t)}= & \dot{S(t)}+\dot{I(t)}-\dot{u(t)} \\
= & a S f_{0}(N)-D_{1} S+b I f_{1}(N)-D_{2} I-u \dot{(t)} \\
= & -D_{1} S-D_{2} I+D u(t) \\
\leq & -\delta W(t), \quad \text { where } \delta=\min \left\{D, D_{1}, D_{2}\right\}>0 .
\end{aligned}
$$

It follows that

$$
W(t) \leq W(0) e^{-\delta t} \rightarrow 0 \text { as } t \rightarrow \infty .
$$

Since $S(t), I(t)$ and $-u(t)$ (since $u(t)<0 \forall t \geq 0$ ) are all nonnegative, this assertions (ii) is true.

With the above lemmas, we can prove the following theorem, showing system (2.1) is dissipative. A system of ordinary differential equations

$$
\frac{d y}{d t}=f(t, y)
$$

defined in a domain $\Omega$ is dissipative if there exists a B such that all solutions $y(t)$ with $y(t) \in \Omega$ for all $t$ satisfy

$$
\limsup _{t \rightarrow \infty}|y(t)| \leq B
$$


Definition of dissipativeness is taken from V. A. Pliss, 'Nonlocal problems of the theory of oscillations' [32].

Theorem 1. System (2.1) with (A2) is dissipative and all solutions initiating in $R_{+}^{3}$ satisfy

$$
0 \leq \liminf u(t)
$$

(ii)

$$
\lim \sup [N(t)+S(t)+I(t)] \leq \frac{D l_{2}^{*}}{\delta}
$$

where $\delta$ is given in the proof of lemma 2.

Proof. The first part of the proof is immediate from lemma 2.

For second part, we note that

$$
\begin{aligned}
\dot{W} & \leq-\delta W(t)+(D-\delta) u(t) \\
& \leq-\delta W(t)+(D-\delta) l_{2}^{*}, \text { since } u(t) \leq N^{*}(t) \leq l_{2}^{*} \text { and } D-\delta \geq 0
\end{aligned}
$$

By variation of constants,

$$
W(t) \leq e^{-\delta t}\left(W(0)-\frac{(D-\delta) l_{2}^{*}}{\delta}\right)+\frac{(D-\delta) l^{*}{ }_{2}}{\delta} .
$$

On the other hand,

$$
N(t)+S(t)+I(t)=W(t)+N^{*}(t) .
$$

Combination of (2.7) and (2.8) proves (ii). Hence the system (2.1) is dissipative.

The following theorems describe extinction of the prey due to lack of nutrient. The conditions given in these theorems describe an environment which provides insufficient nutrient for prey survival and consequently they are washed away from the system.

Theorem 2. Suppose (A2) holds.

i) If

$$
\sigma_{0}=\frac{1}{\omega} \int_{0}^{\omega}\left[a f_{0}\left(N^{*}(t)\right)-D_{1}\right] d t<0
$$

then $S(t)$ tends to zero exponentially, and

ii) if

$$
\sigma_{1}=\frac{1}{\omega} \int_{0}^{\omega}\left[b f_{1}\left(N^{*}(t)\right)+\lambda-D_{2}\right] d t<0,
$$

then $I(t)$ tends to zero exponentially.

Proof. The result (ii) of lemma 2 is sufficient to show the theorem is valid.

So our assumption that $u(t) \geq 0$ implies $N^{*}(t) \geq N(t)$ for $t \geq t_{1} \geq 0$.

Since $f_{0}$ is increasing, so

$$
f_{0}(N(t)) \leq f_{0}\left(N^{*}(t)\right) \text { for } t \geq t_{1}
$$




$$
\frac{d S}{d t}=a S f_{0}(N)-\lambda \frac{S I}{S+I}-D_{1} S \leq S\left(a f_{0}\left(N^{*}\right)-D_{1}\right) \text { for } t \geq t_{1}
$$

We have

$$
\begin{aligned}
0 \leq S(t) & \leq S(0) e^{\int_{0}^{t_{1}}\left[a f_{0}\left(N^{*}(t)\right)-D_{1}\right] d t} e^{\int_{t_{1}}^{t_{1}+n \omega}\left[a f_{0}\left(N^{*}(t)\right)-D_{1}\right] d t} \\
& =S(0) e^{\int_{0}^{t_{1}}\left[a f_{0}\left(N^{*}(t)\right)-D_{1}\right] d t} e^{\int_{0}^{n \omega}\left[a f_{0}\left(N^{*}(t)\right)-D_{1}\right] d t}
\end{aligned}
$$

for some $n=n(t)>0$.

If we take

$$
\sigma_{0}<0 \text { i.e. } \omega \sigma_{0}=\int_{0}^{\omega}\left[a f_{0}\left(N^{*}(t)\right)-D_{1}\right] d t<0
$$

then (2.9) implies that $S(t)$ tends to zero exponentially. Similarly we can proof that $I(t)$ tends to zero exponentially provided the conditions stated in the theorem is satisfied. Hence the theorem.

Remark 1. If $\sigma_{0}<0$ and $\sigma_{1}<0$ then for every solution $(N(t), S(t), I(t))$ of (2.1), $S(t)$ and $I(t)$ both tend to zero exponentially. If $\sigma_{0}>0$ and $\sigma_{1}>0$ then for every solution $(N(t), S(t), I(t))$ of (1), $S(t)$ and $I(t)$ both co-exist.

Theorem 3. Let us suppose that $a>D_{1}$ and $b>D_{2}$, then (A2) implies that there exists unique $\lambda_{0}$ and $\lambda_{1}$ for which $f_{0}\left(\lambda_{0}\right)=\frac{D_{1}}{a}$ and $f_{1}\left(\lambda_{1}\right)=\frac{D_{2}-\lambda}{b}$. Moreover we assume that the derivative $f_{0}^{\prime}$ of $f_{0}$ and $f_{1}^{\prime}$ of $f_{1}$ are strictly decreasing.

i) If

$$
\lambda_{0} \geq \hat{N}
$$

then $S(t)$ tends to zero exponentially as $t \rightarrow \infty$, and

ii) if

$$
\lambda_{1} \geq \hat{N}
$$

then $I(t)$ tends to zero exponentially as $t \rightarrow \infty$.

Proof. Denote

$$
J_{+}=\left\{t \in[0, \omega]: N^{*}(t) \geq \lambda_{0}\right\} \text { and } J_{-}=[0, \omega] \backslash J_{+}
$$

is the compliment of $J_{+}$in $[0, \omega] . J_{-}$is a nonempty open set in $[0, \omega]$.

By the mean value theorem,

$$
f_{0}\left(N^{*}(t)\right)-f_{0}\left(\lambda_{0}\right)=f_{0}^{\prime}(\theta(t))\left(N^{*}(t)-\lambda_{0}\right),
$$

where $\theta(t)$ lies between $\lambda_{0}$ and $N^{*}(t)$.

Thus

$$
f_{0}\left(N^{*}(t)\right)-f_{0}\left(\lambda_{0}\right) \leq f_{0}^{\prime}\left(\lambda_{0}\right)\left(N^{*}(t)-\lambda_{0}\right) .
$$

If $t \in J_{+}$and $\theta(t) \geq \lambda_{0}$ then $f_{0}^{\prime}(\theta(t)) \leq f_{0}^{\prime}\left(\lambda_{0}\right)$ as $f_{0}^{\prime}$ is strictly decreasing. But if $t \in J_{-}$and $\theta(t)<\lambda_{0}$ then $f_{0}^{\prime}(\theta(t))>f_{0}^{\prime}\left(\lambda_{0}\right)$.

Hence in both cases the above inequality holds. 
From the inequality (2.10) and nonemptiness of $J_{-}$, we get

$$
\int_{0}^{\omega}\left[f_{0}\left(N^{*}(t)\right)-f_{0}\left(\lambda_{0}\right)\right] d t<\omega f_{0}^{\prime}\left(\lambda_{0}\right)\left(\hat{N}-\lambda_{0}\right) .
$$

Therefore, we have

$$
\sigma_{0}=\frac{a}{\omega} \int_{0}^{\omega}\left[f_{0}\left(N^{*}(t)\right)-f_{0}\left(\lambda_{0}\right)\right] d t<a f_{0}^{\prime}\left(\lambda_{0}\right)\left(\hat{N}-\lambda_{0}\right) \leq 0, \text { if } \hat{N} \leq \lambda_{0} .
$$

This completes the first part (i) of the theorem. The second part of (ii) follows the similar proof and hence omitted.

Hence the theorem.

Remark 2. If we take $f_{0}(N)=\frac{N}{k_{1}+N}$ then $f_{0}\left(\lambda_{0}\right)=\frac{D_{1}}{a}$ implies $\lambda_{0}=\frac{k_{1} D_{1}}{a-D_{1}}$ and condition $\lambda_{0} \geq \hat{N}$ takes the form $\frac{k_{1} D_{1}}{a-D_{1}} \geq \hat{N}$. If we take $f_{1}(N)=\frac{N}{k_{2}+N}$ then $f_{1}\left(\lambda_{1}\right)=\frac{D_{2}-\lambda}{b}$ implies $\lambda_{1}=\frac{k_{2}\left(D_{2}-\lambda\right)}{b-D_{2}+\lambda}$ and condition $\lambda_{1} \geq \hat{N}$ takes the form $\frac{k_{2}\left(D_{2}-\lambda\right)}{b-D_{2}+\lambda} \geq \hat{N}$.

We shall now summarize our analytical findings in a tabular form (Table 1).

Table 1: Table summarizing the analytical findings of system (2.1).

\begin{tabular}{|c|c|}
\hline Threshold conditions & Dynamical behaviour \\
\hline$\sigma_{0}<0$ & Extinction of susceptible phytoplankton \\
\hline$\sigma_{1}<0$ & Extinction of infected phytoplankton \\
\hline$\sigma_{0}>0 \& \sigma_{1}>0$ & Co-existing of both phytoplanktons \\
\hline
\end{tabular}

\section{Infected population is incapable of nutrient consumption}

The study of Uhlig and Sahling [13] on the dinolagellate Noctiluca scintillans in the German Bight showed that infected phytoplankton cells do not feed any more and are also not in a position to reproduce. Under this assumption the system (2.1) takes the following form:

$$
\begin{aligned}
\frac{d N}{d t} & =D\left(N^{0}(t)-N\right)-a S f_{0}(N) \\
\frac{d S}{d t} & =a S f_{0}(N)-\lambda \frac{S I}{S+I}-D_{1} S \\
\frac{d I}{d t} & =\lambda \frac{S I}{S+I}-D_{2} I
\end{aligned}
$$

The following theorem describes the extinction of both phytoplanktons due to lack of nutrient. 
Theorem 4. Suppose (A2) holds.

i) If

$$
\sigma_{0}=\frac{1}{\omega} \int_{0}^{\omega}\left[a f_{0}\left(N^{*}(t)\right)-D_{1}\right] d t<0
$$

then $S(t)$ tends to zero exponentially as $t \rightarrow \infty$, and

ii) if

$$
\sigma_{1}^{*}=\lambda-D_{2}<0,
$$

then $I(t)$ tends to zero exponentially as $t \rightarrow \infty$.

Proof.

Proof follows from the Theorem 2 by putting $b=0$

Remark 3. If $\sigma_{0}<0$ and $\sigma_{1}^{*}<0$ then for every solution $(N(t), S(t), I(t))$ of (3.1) $S(t)$ and $I(t)$ both tend to zero exponentially. If $\sigma_{0}>0$ and $\sigma_{1}^{*}>0$ then for every solution $(N(t), S(t), I(t))$ of (3.1) $S(t)$ and $I(t)$ both coexist.

Theorem 5. Suppose (A2) holds and the derivative $f_{0}^{\prime}$ of $f_{0}$ is strictly decreasing. If $\lambda_{0} \geq \hat{N}$ then $S(t)$ tends to zero exponentially as $t \rightarrow \infty$.

Proof. Similar to theorem 3.

Now, we summarize the results of system (3.1) in the following table (Table2)

Table 2: Table is summarizing the analytical findings of model (3.1).

\begin{tabular}{|c|c|}
\hline Threshold conditions & Dynamical behaviour \\
\hline$\sigma_{0}<0$ & Extinction of susceptible phytoplankton \\
\hline$\sigma_{1}^{*}<0$ & Extinction of infected phytoplankton \\
\hline$\sigma_{0}>0 \& \sigma_{1}^{*}>0$ & Co-existing of both phytoplanktons \\
\hline
\end{tabular}

\section{Numerical Simulations}

In numerical experiment we consider the following forms for nutrient uptake $f_{0}(N)=\frac{N}{k_{1}+N}$ and $f_{1}(N)=\frac{N}{k_{2}+N}$ with $k_{1}$ and $k_{2}$ are half-saturation constants. The general system $(2.1)$ reduces to

$$
\begin{aligned}
\frac{d N}{d t} & =D\left(N^{0}(t)-N\right)-a \frac{S N}{k_{1}+N}-b \frac{I N}{k_{2}+N} \\
\frac{d S}{d t} & =a \frac{S N}{k_{1}+N}-\lambda \frac{S I}{S+I}-D_{1} S \\
\frac{d I}{d t} & =b \frac{I N}{k_{2}+N}+\lambda \frac{S I}{S+I}-D_{2} I
\end{aligned}
$$


where

$$
N^{0}(t)=0.5+A \sin (\omega t) .
$$

We perform our numerical experiments by considering the following three different situation of the system (4.1).

Case 0: Both phytoplanktons are able to consume nutrient (i.e. we consider in full system (4.1)).

Case I: The infected phytoplankton is not able to consume nutrient (i.e. the system (4.1) with $b=0)$.

Case II: Nutrient input is constant (i.e. the system (4.1) with $N^{0}=$ const).

For numerical simulations we use the following hypothetical parameter values and simulations have been carried out with MATLAB.

$$
\begin{array}{r}
D=0.0012, D_{1}=0.02, D_{2}=0.05, a=1.5, b=0.3 \\
k_{1}=0.1, k_{2}=0.2, \lambda=0.2, A=0.5, \omega=0.19
\end{array}
$$

Numerical simulations have been carried out with the help of MATLAB software.

Table 3: Table summarizing the analytical findings in light of numerical values given in (4.2) for the system (2.1).

\begin{tabular}{|c|c|c|c|}
\hline $\begin{array}{c}\text { Threshold } \\
\text { conditions }\end{array}$ & $\begin{array}{c}\text { Variable } \\
\text { parameter } \\
\lambda\end{array}$ & $\begin{array}{c}\text { Dynamical } \\
\text { behaviour }\end{array}$ & Figure \\
\hline$\sigma_{0}=-0.0158984<0$ & $\lambda=0.39$ & $\begin{array}{c}\text { Extinction of susceptible } \\
\text { phytoplankton }\end{array}$ & Fig. 5 \\
\hline$\sigma_{1}=-0.00958928<0$ & $\lambda=0.04$ & $\begin{array}{c}\text { Extinction of } \\
\text { infected phytoplankton }\end{array}$ & Fig. 1a \\
\hline$\sigma_{0}=0.4288>0 \& \sigma_{1}=0.302775>0$ & $\lambda=0.3$ & $\begin{array}{c}\text { Co-existing of both } \\
\text { phytoplankton populations }\end{array}$ & Fig. 5 \\
\hline
\end{tabular}

In our simulation experiments we like to observe the effect of nutrient-concentration and disease transmission factor in the dynamics of $N-S-I$ systems described in Case 0-Case II of the system (4.1). Before that we present the following table (Table3) to verify the analytical findings in light of numerical observations. Now we observe the following steps: (i) in system (4.1) we vary the force of infection $\lambda$ (ii) in the subsequent stages, we compare the observations thus obtained for the system (4.1) with $b=0$ (i.e. Case I) and system (4.1) with $N(t)=$ const (i.e. Case II) and (iii) finally we observe the rate of nutrient concentration in the dynamics of system (4.1) and system (4.1) with $b=0$. The dynamics of the system (4.1) with three different cases (viz Case 0, Case I and Case II) have observed by considering the force of infection $\lambda$ as a bifurcation parameter.

The infected phytoplankton population does not persist below a minimum threshold of infection $\left(\lambda \leq \lambda_{\text {mim }}=0.04\right)$ in the system(4.1) and in Case II of system(4.1) (see Fig. 1a,c). But 


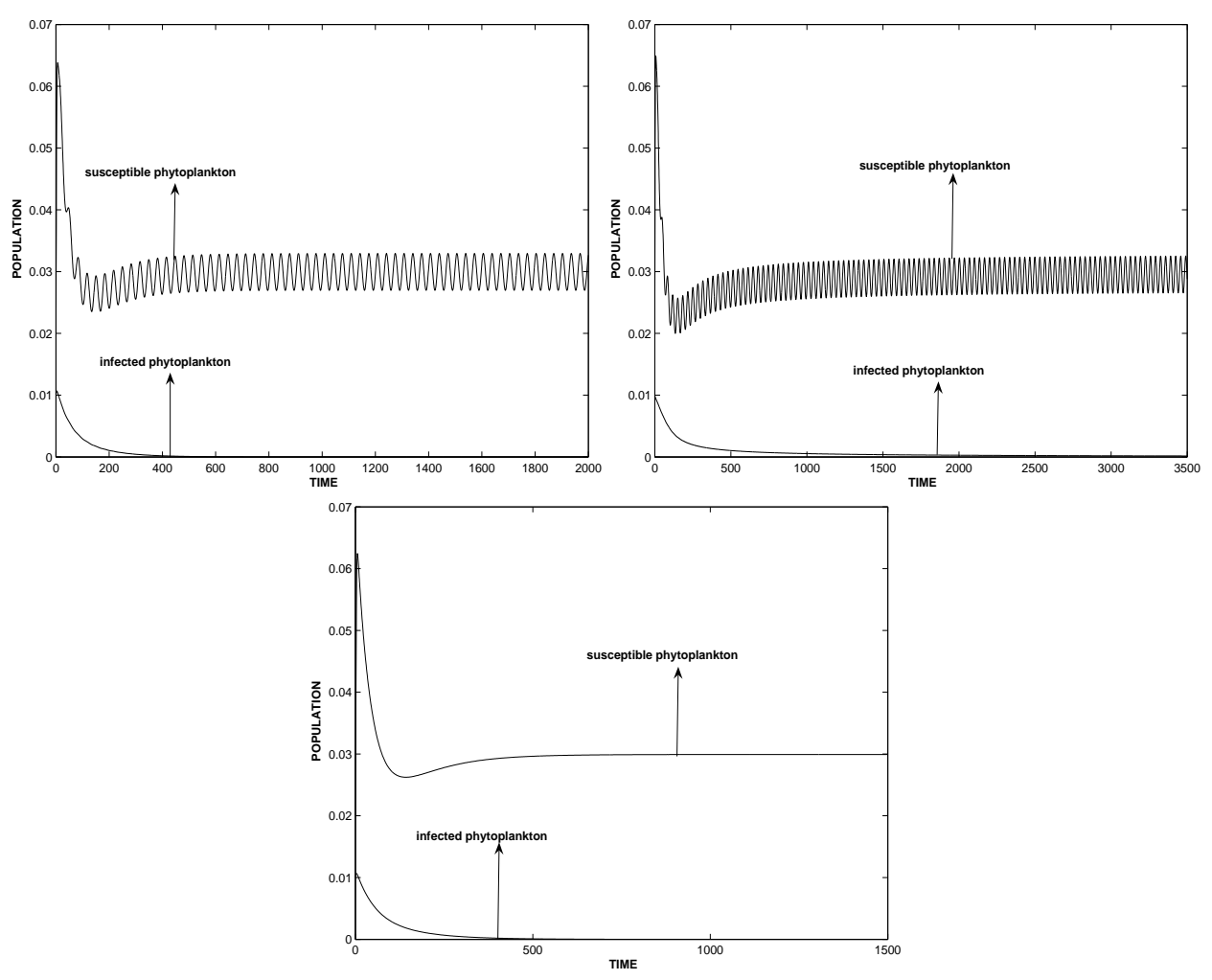

Figure 1: Figures(a) and (c) show the extinction of infected phytoplankton for $\lambda=0.04$ in Case 0 and Case II of the system (4.1) respectively and (b) shows the extinction of infected phytoplankton for $\lambda=0.05$ in Case I keeping other parameters fixed as given in (4.2).
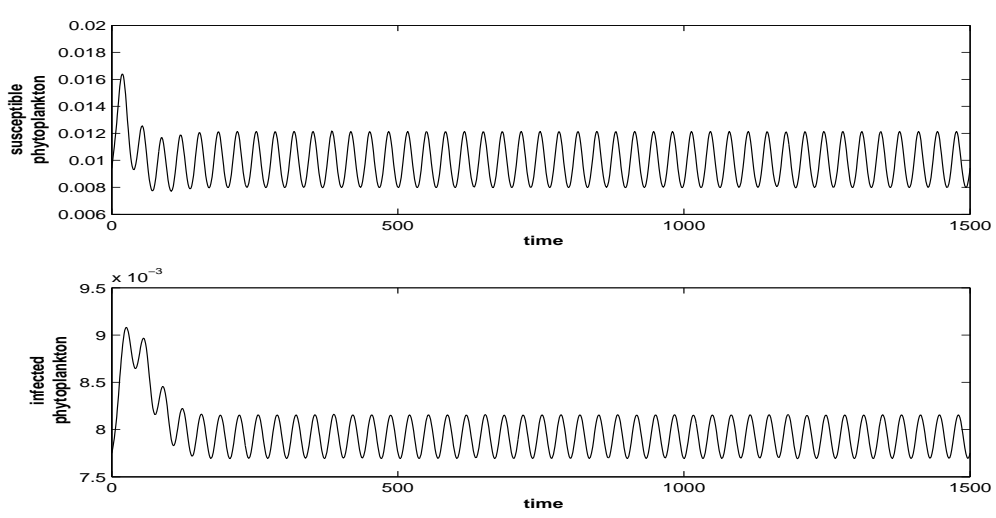

Figure 2: Figure shows that both phytoplanktons coexist in limit cycle oscillating position for $\lambda=0.05$ in the system (4.1), keeping other parameters fixed as given in (4.2). 
Table 4: Table summarizing the analytical findings in light of numerical values given in (4.2) for the system (3.1).

\begin{tabular}{|c|c|c|c|}
\hline $\begin{array}{c}\text { Threshold } \\
\text { conditions }\end{array}$ & $\begin{array}{c}\text { Variable } \\
\text { parameter } \\
\lambda\end{array}$ & $\begin{array}{c}\text { Dynamical } \\
\text { behaviour }\end{array}$ & Figure \\
\hline$\sigma_{1}=-0.01<0$ & $\lambda=0.05$ & $\begin{array}{c}\text { Extinction of } \\
\text { infected phytoplankton }\end{array}$ & Fig1b \\
\hline$\sigma_{0}=0.25572>0 \& \sigma_{1}=0.34>0$ & $\lambda=0.39$ & $\begin{array}{c}\text { Co-existing of both } \\
\text { phytoplankton populations }\end{array}$ & Fig6 \\
\hline
\end{tabular}
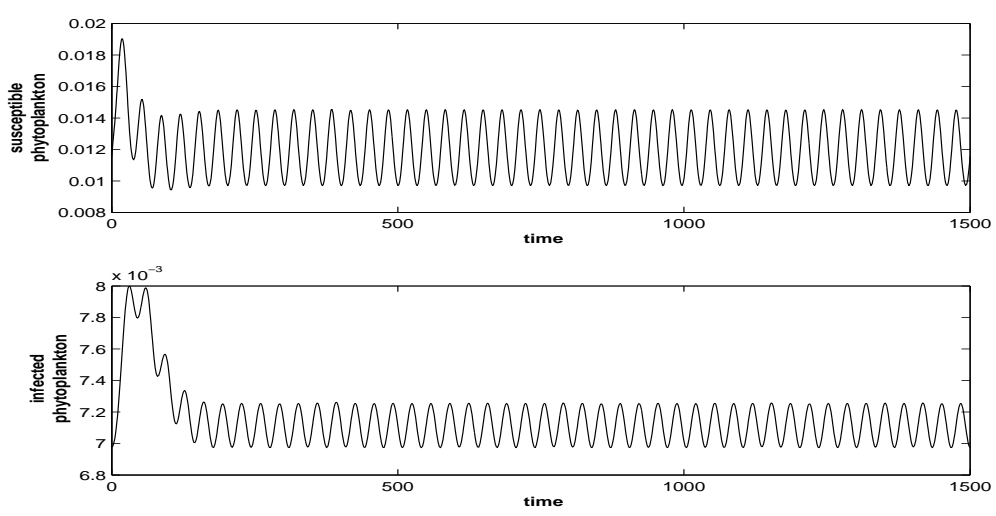

Figure 3: Figure shows that both phytoplanktons coexist in limit cycle oscillating position for $\lambda=0.06$ in Case I of the system (4.1), keeping other parameters fixed as given in (4.2).

we also notice that the infected phytoplankton does not survive below a minimum threshold of infection $\left(\lambda \leq \lambda_{\text {min }}=0.05\right)$ in Case I of the system (4.1)(see Fig. 1b). We observe that both susceptible and infected phytoplanktons coexist in oscillatory (limit cycle) position for $\lambda=0.05$ in Case 0 and for $\lambda=0.06$ in case I of the system (4.1) respectively (see Figs. 2 and 3). But in case II of the system (4.1) both phytoplanktons coexist in stable situation for $\lambda=0.05$ (see Fig. 4). It is clearly found that both phytoplanktons coexist in limit cycle oscillation for $0.05 \leq \lambda \leq 0.22$ and both phytoplanktons coexist in chaotic position for $0.22<\lambda<0.39$ in Case 0 but in Case I both phytoplanktons coexist in limit cycle oscillation for $0.06 \leq \lambda \leq 0.22$ and chaotic position for $0.22<\lambda$ (see Figs. 2 and 3, and Figs. 5 and 6). It is also found that susceptible phytoplankton does not survive for $\lambda \geq 0.39$ in Case 0 but both phytoplankton persist in chaotic position for $\lambda \geq 0.39$ in Case I (see Figs. 5 and 6).

It is noticed that as $\lambda$ increases, the system (4.1) enters into a chaotic region from limit cycle oscillation and a further increase in the value of $\lambda$ leads to the extinction of the susceptible phytoplankton population, (see Fig. 5). On the other hand, when infected phytoplankton is not in a state to consume nutrient (the dynamics presented in the Case I of the system (4.1)), both susceptible and infected phytoplankton settle down into chaotic region from limit cycle oscillation and there is 


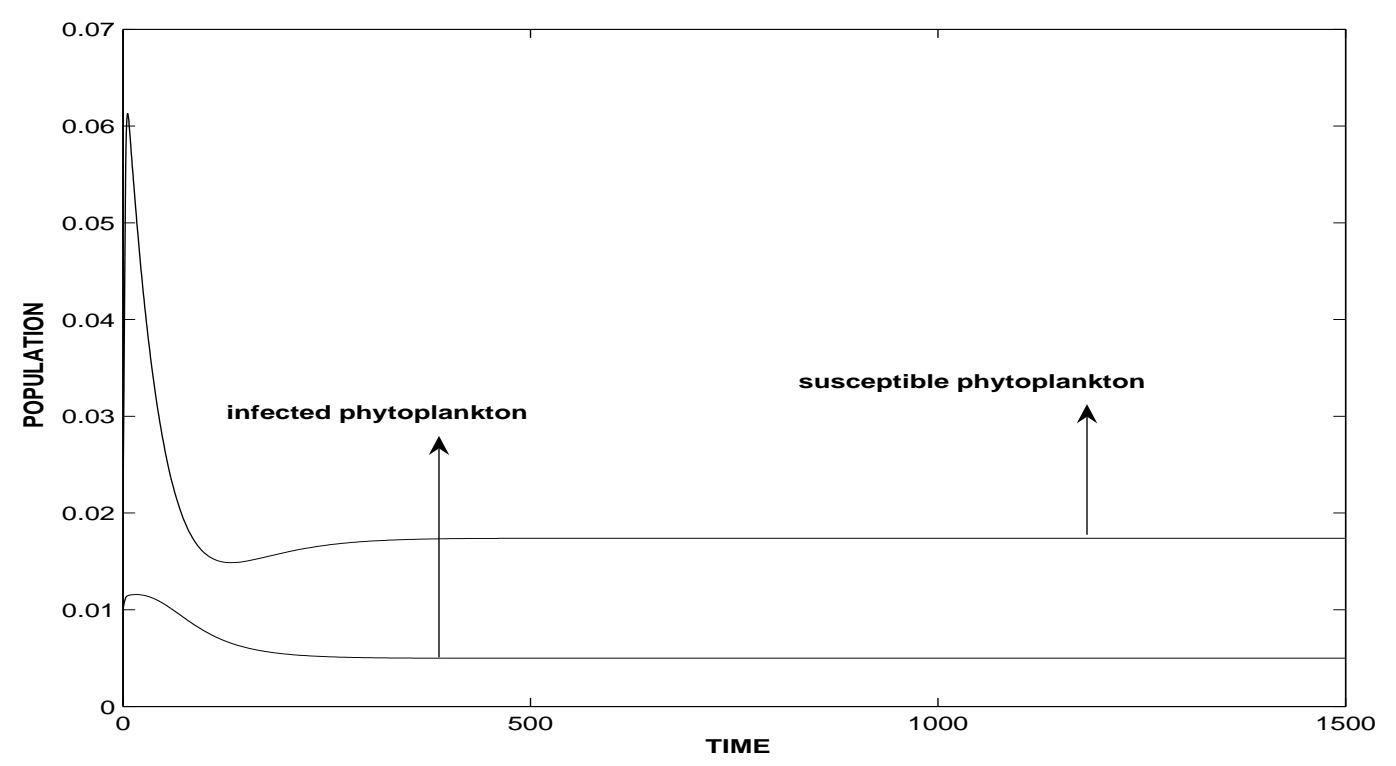

Figure 4: Figure shows that both phytoplanktons coexist in steady stable position for $\lambda=0.05$ in Case II of the system (4.1), keeping other parameters fixed as given in (4.2).

no chance of extinction of susceptible phytoplankton (see Fig. 6). It is clear from the above observations that susceptible phytoplankton does not survive in Case 0 for higher values of $\lambda$ whereas both phytoplankton survive for higher values of $\lambda$ for Case I. This result indicates that for the co-existence of the phytoplankton, incapability of nutrient consumption by infected phytoplankton play an important role. In this connection we like to mention the experimental observations of Uhlig and Sahling [13] on dinoflagellate Noctiluca scintillans (milliaris). They observed that Noctiluca scintillans in German Bight showed clear isolated bursts occured fairly regularly when infected Noctiluca scintillans did not feed anymore.

From Figs. 4 and 7 we observe that both phytoplankton coexist in stable position for $0.05 \leq$ $\lambda \leq 0.22$ and limit cycle position for $0.22<\lambda<0.39$ and susceptible phytoplankton wipes out for $\lambda \geq 0.39$ in Case II. For Case II we observe that both phytoplanktons persist in limit cycle oscillation for $\lambda>0.22$ when infected phytoplankton is unable to consume nutrient (see Fig. 8).

It is observed for Case II of system (4.1), both susceptible and infected population enter into limit cycle oscillation from steady state and ultimately the susceptible phytoplankton goes to extinction for variation of $\lambda$ (see Fig. 7). In such case, if the infected population does not consume nutrient, all population co-exist (see Fig. 8). Chattopadhyay et al. [15] also observed both phytoplanktons do not persist when both phytoplanktons are able to consume nutrient whereas both phytoplankton coexist if infected phytoplankton do not consume nutrient. Our analytical and numerical analysis suggest that inconsumption of nutrient by infected phytoplankton is a crucial factor for coexistence of both phytoplanktons species.

To observe the role of seasonal forcing strength $(A)$ and force of infection $(\lambda)$ in the Case 0 and Case I of equations (4.1) we plot 2-D parameter figure. We observe that when infected phytoplankton consume nutrient there is a chance of extinction of susceptible phytoplankton for higher 

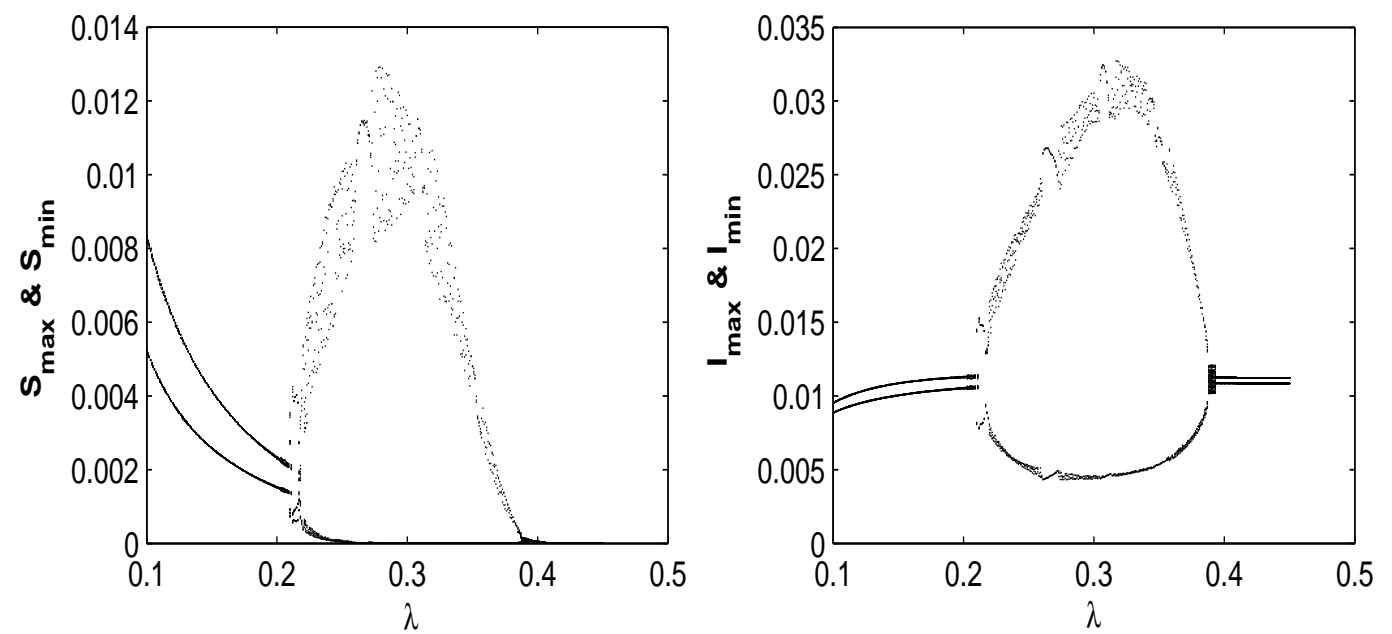

Figure 5: Figure shows that the bifurcation diagram for $\lambda \in[0.1,0.5]$ and extinction of susceptible phytoplankton for the system (4.1), keeping other parameters fixed as given in (4.2).
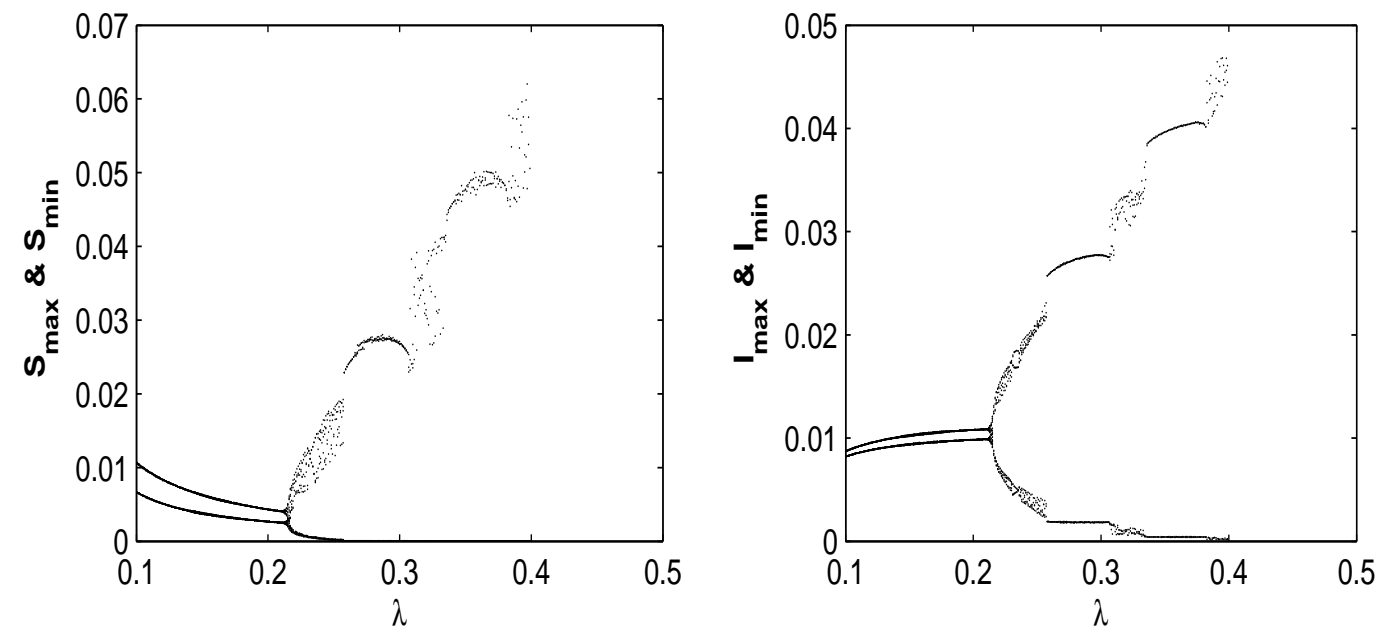

Figure 6: Figure shows the co-existing of all population for Case I of the system (4.1) (all the other parameter values fixed as given in (4.2). 

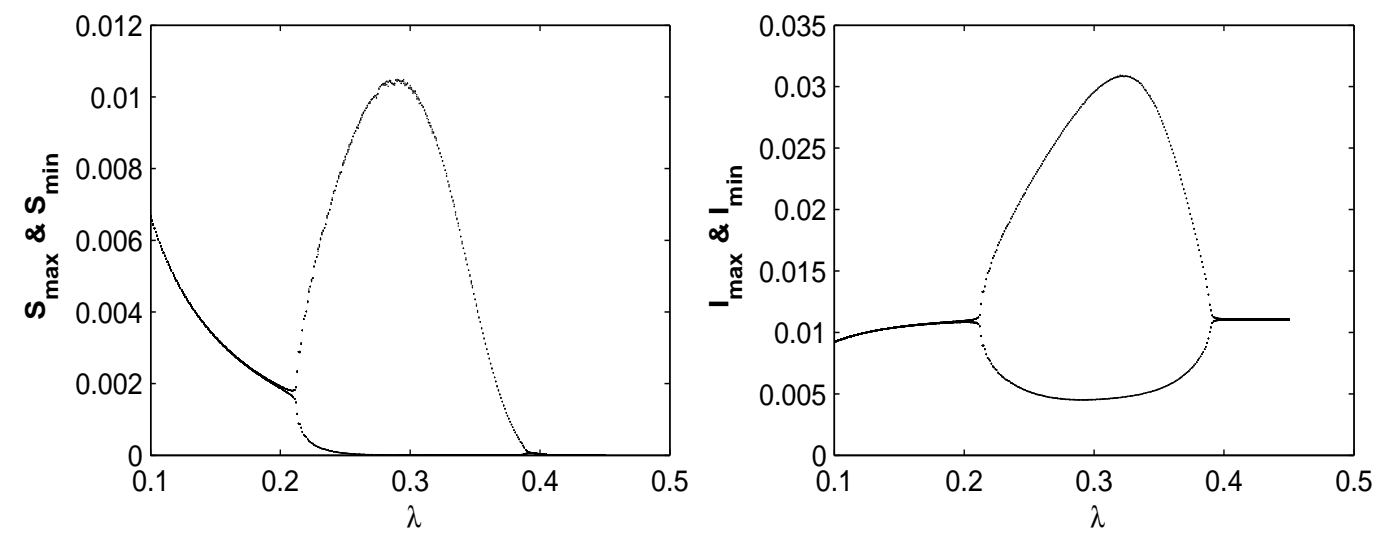

Figure 7: Figure shows the bifurcation diagram for $\lambda \in[0.1,0.5]$ and the extinction of susceptible phytoplankton for Case II of the system (4.1) (all the other parameter values fixed as given in (4.2).
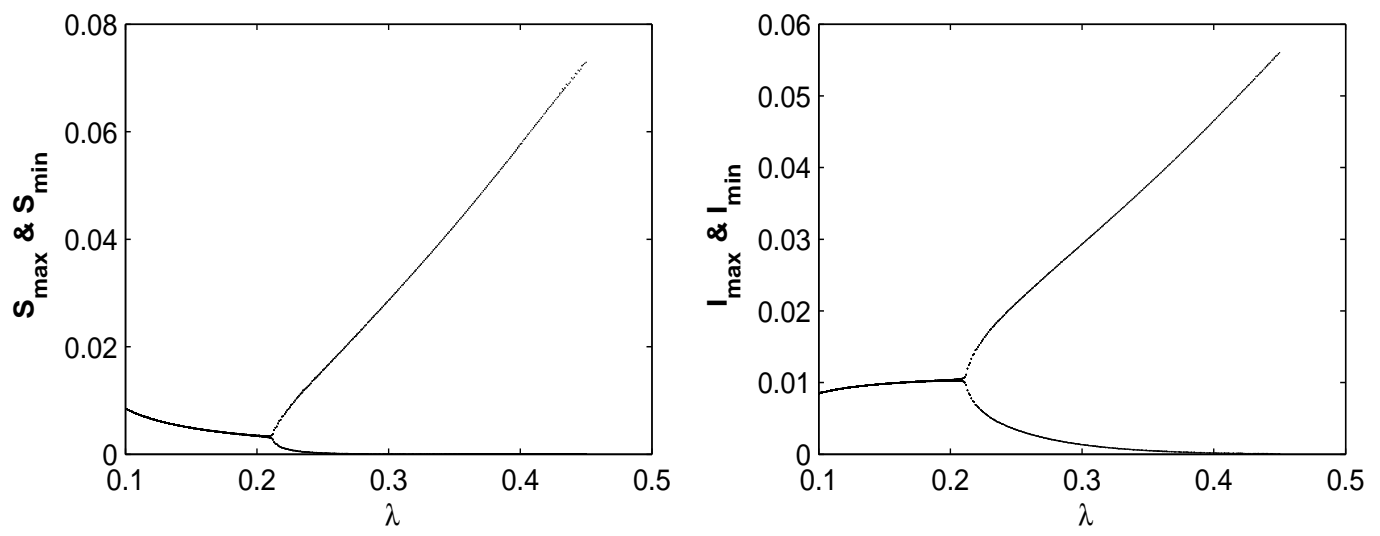

Figure 8: Figure shows co-existing of all species when infected population is not able to consume nutrient for Case II of the system (4.1) (all the other parameter values fixed as given in (4.2).) 


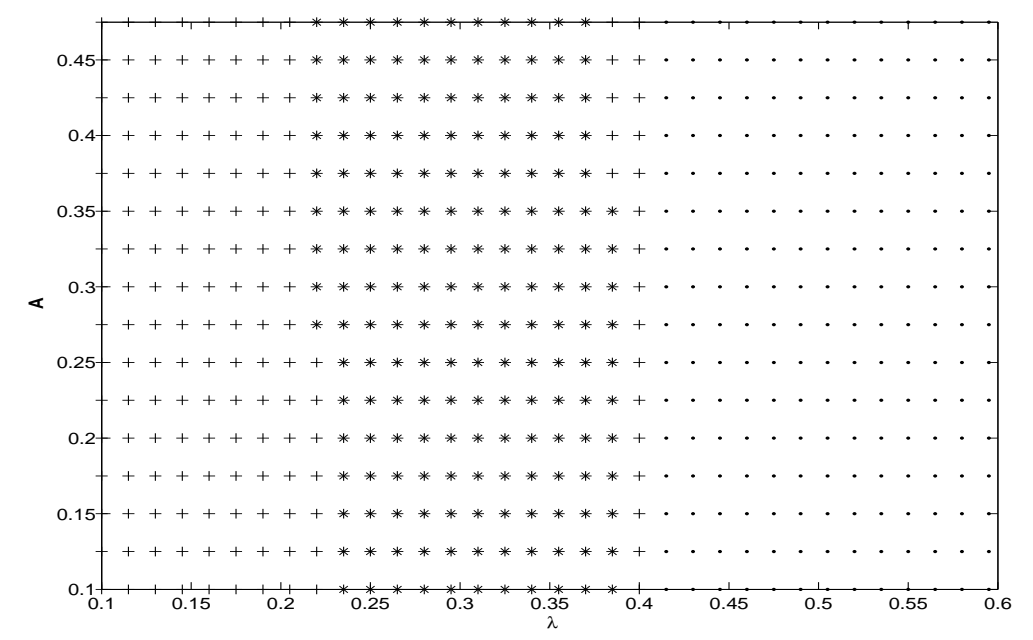

Figure 9: Figure shows the plot between seasonal forcing strength $A$ and force of infection $\lambda$ for the equation (4.1) (i.e.Case 0). ' + ' represents the periodic oscillation, ' $*$ ' represents the aperiodic oscillation and ' ' represents extinction of the susceptible population. The parameter values are given in (4.2)

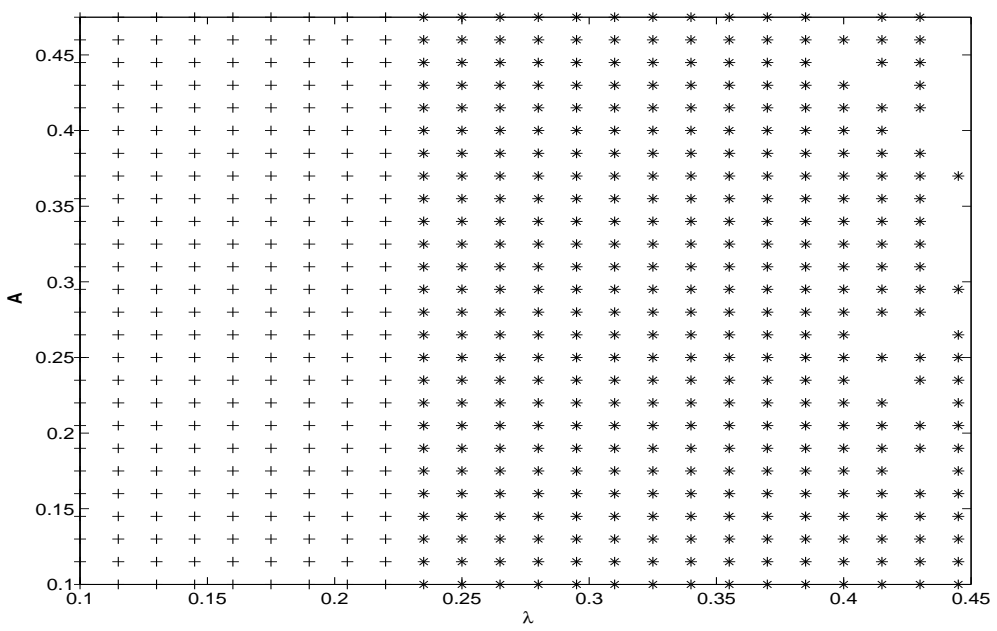

Figure 10: Figure shows the plot between seasonal forcing strength $A$ and force of infection $\lambda$ for Case I of the equation (4.1). ' + ' represents the periodic oscillation and ' $*$ ' represents the aperiodic oscillation. The parameter values are given in (4.2). 
values of $\lambda$ but when the infected phytoplankton do not consume nutrients, then the susceptible phytoplankton will never be washed away from the system for higher values of $\lambda$. We observe for both the system that when the values of $\lambda$ and $A$ is small the systems show periodic oscillation, see ' + ' in Figs. 9 and 10. But for higher values of $\lambda$ or $A$ we observe that the system shows aperiodic oscillation, see ' $*$ ' in Figs. 9 and 10. Finally for large values of $\lambda$ we observe that the susceptible phytoplankton goes to extinction when the infected phytoplankton consume nutrients, see '? in Fig. 9.

For convenience, we summarize the above findings in a tabular form (Table 5).

Table 5: Behaviour of system(4.1).

\begin{tabular}{|c|c|c|c|}
\hline $\begin{array}{c}\text { Variation of } \\
\lambda\end{array}$ & Case 0 & Case I & Case II \\
\hline$\lambda \leq 0.04$ & $\begin{array}{l}\text { Extinction of } \\
\text { infected } \\
\text { phytoplankton }\end{array}$ & $\begin{array}{l}\text { Extinction of } \\
\text { infected } \\
\text { phytoplankton }\end{array}$ & $\begin{array}{l}\text { Extinction of } \\
\text { infected } \\
\text { phytoplankton }\end{array}$ \\
\hline $0.05=\lambda$ & Limit cycle & $\begin{array}{l}\text { Extinction of infected } \\
\text { phytoplankton }\end{array}$ & $\begin{array}{l}\text { Both phytoplanktons } \\
\text { coexist in steady state } \\
\text { stable position }\end{array}$ \\
\hline $0.06 \leq \lambda \leq 0.22$ & Limit cycle & Limit cycle & $\begin{array}{l}\text { Both phytoplanktons } \\
\text { coexist in steady state } \\
\text { stable position }\end{array}$ \\
\hline $0.22<\lambda<0.39$ & Chaotic oscillation & Chaotic oscillation & Limit cycle \\
\hline$\lambda \geq 0.39$ & $\begin{array}{l}\text { Extinction of } \\
\text { susceptible } \\
\text { phytoplankton }\end{array}$ & $\begin{array}{l}\text { co-existing of both } \\
\text { phytoplanktons }\end{array}$ & $\begin{array}{l}\text { Extinction of } \\
\text { susceptible } \\
\text { phytoplankton }\end{array}$ \\
\hline
\end{tabular}

\section{Concluding Remarks}

In marine ecosystem two factors viz seasonality of nutrient and viral disease in phytoplankton influence the dynamics of plankton. However, viral disease in phytoplankton affects significantly the dynamics of nutrient-plankton model in the presence of periodic nutrient input. In this paper we consider an $N P$ model with periodic nutrient input and compare it with different cases arising due 
to constant nutrient input and non-consumption nutrient by infected phytoplankton. We begin with a general model where we took a general functional response and a general periodic function for the nutrient input. The analytical results corresponding to the general model leads to two distinct thresholds $\sigma_{0}$, and $\sigma_{1}$. The existence of susceptible and infected phytoplankton, the endemicity of the system and disease freeness of the system have been determined by these thresholds. When both phytoplankton uptake nutrient, the system is endemic for $\sigma_{1} \geq 0$ and disease free for $\sigma_{1}<0$ and both plankton co-exist for $\sigma_{0}>0$ and $\sigma_{1}>0$. But endemicity of system is determined by $\sigma_{1}^{*}$ (it is obtained by putting $b=0$ in $\sigma_{1}$ ) and $\sigma_{0}>0$ and $\sigma_{1}^{*}>0$ implies the persistence of susceptible and infected phytoplankton when infected phytoplankton is incapable of uptaking nutrient. The numerical simulations in our study demonstrates a number of interesting phenomena. With the increase of the force of infection, the system with periodic nutrient input goes to chaotic region from limit cycle while the system with constant nutrient goes to limit cycle from stable position. In this connection we like to mention the result of Inoue and Kamifukumoto [26] that external forcing can induce oscillations in a simple prey-predator system. Ecological communities are generally embeded in periodically varying environments. It has been shown by Rinaldi et al. [30] that such periodic external forcing may lead to a classical prey-predator system into limit cycle oscillation, period doubling and chaos. We claim from the observations of our proposed model with periodic nutrient input and constant nutrient input that seasonal forcing is responsible for chaotic dynamics. In this connection we like to mention that Huppert et al. [2] extended the basic $N P$ model by introducing periodic seasonal forcing which induced all kinds of complex dynamics from limit-cycles to chaos. We also observe that when the force of infection is high, the susceptible phytoplankton co-exist with infected phytoplankton only if infected phytoplankton is incapable of uptaking nutrient. This observation is also supported by the experimental observation of Uhlig and Sahling [13].

We finally observe from the 2-D parameter figure that for low values of $\lambda$ and $A$ the systems show periodic solution while for high values they show aperiodic solution, which may lead to the extinction of the phytoplankton population if the infected phytoplankton population consumes nutrient.

Acknowledgement: The authors are grateful to the learned reviewers for their critical comments and suggestions on the earlier version of the paper.

\section{References}

[1] A. Bouma, M.C.M. De Jong, T.G. Kimman. Transmission of pseudorabies virus within pig populations is independent of the size of the population. Preventative Veterinary Medicine, 23 (1995), 163-72.

[2] A. Huppert, B. Blasius, R. Olinky, L. Stone. A model for seasonal phytoplankton blooms. J. Theo. Biol. 236 (2005), 276-290. 
[3] A.J. Taylor. Characteristic properties of model for the vertical distribution of phytoplankton under stratification. Ecol. Model. 40 (1988), 175-199.

[4] B.W. Frost. Grazing control of phytoplankton stock in the open sub-arctic pacific Ocean: a model assessing the of mesozooplankton, particularly the large calanoid copepod neocalanus. Mar. Ecol. Ser. 39 (1987), 49-68.

[5] C.P.D. Brussaard, R.S. Kempers, A.J. Kop, R. Tiegman, M. Heldel. Virus like particles in a summer bloom of Emiliania huxleyi in the North Sea. Aq. Microbial. Ecol. 10 (1996), 105-113.

[6] C. Suttle, A. Charm, M. Cottrell. Infection of phytoplankton by viruses and reduction of primary productivity. Nature 347 (1990), 467-469.

[7] C.A. Suttle, A.M. Chan. Marine cyanophages infecting oceanic a nd coastal strains of Synechococcus: abundance, morphology, cross-infectivity and growth characteristics. Mar. Ecol. Prog. Ser. 92 (1993), 99-109.

[8] D.M.C.M. Jong, O. Diekmann, J.A.P. Heesterbeek. How does transmission depend on population size? in Human Infectious Disease, Epidemic Models, D.Mollisison, ed., Cambridge University press, Cambridge, UK, (1995) 84-94.

[9] E. Beltrami and T.O. Carroll. Modeling the role of viral disease in recurrent phytoplankton blooms. J. Math. Biol. 32 (1994), 857-863.

[10] G.J. Butler, P. Waltman. Persistence in dynamical systems. J. Diff. Equations 63 (1986), 255263.

[11] G. Bratbak, M. Leavasseur, S. Michand, G. Cantin, E. Fernandez, M. Heldel. Viral activity in relation to Emiliania huaxleyi blooms: a mechanism of DMSP release ? Mar. Ecol. Progr. Ser. 128 (1995), 133-142.

[12] G.T. Evans, J.S. Parslow. A model of annual plankton cycles. Bio. Oceanogr. 3 (1985), $327-$ 427.

[13] G. Uhlig, G. Sahling. Long-term studies on Noctiluca scintillans in the German Bight. Neth. J. Sea Res. 25 (1992), 101-112.

[14] H.W. Hethcote. The mathematics of infectious disease. SIAM Rev., 42 (2000), 599-653.

[15] J. Chattopadhyay, R.R. Sarkar, S. Pal. Dynamics of nutrient-phytoplankton interaction in the presence of viral infection. BioSystems, 68 (2003), 5-17 .

[16] J.A. Fuhrman. Marine viruses and their biogeochemical and ecological effects. Nature 399 (1999), 541-548.

[17] J.A. Mayer and F.J.R. Taylor. A virus which lyses the marine nanoflagellate micromonas pusilla. Nature, 281 (1979), 299-301 . 
[18] J. S. Wroblewski, J. L. Sarmiento, G. R. Flierl. An ocean basin scale model of plankton dynamics in the North Atlantic, 1, solutions for the climatological oceanographic condition in May. Global Biogeochem. Cycles 2 (1988), 199-218.

[19] K.P. Hadeler, H.I. Freedman. Predator-prey population with parasitic infection. J. Math. Biol. 27 (1989), 609-631.

[20] K. Nagasaki, M.Yamaguchi. Isolation of a virus infectious to the harmful bloom causing microalga Heterosigma akashiwo (Raphidophyceae). Aquat. Microbial. Ecol. 13 (1997), 135140.

[21] K. Tarutani, K. Nagasaki, M.Yamaguchi. Viral impacts on total abundance and clonal composition of the harmful bloom-forming phytoplankton Heterosigma akashiwo. Appl. Environ. Microbial. 66 (2000), No. 11, 4916-4920.

[22] K.E. Wommack, R.R. Colwell. Virioplankton: viruses in aquatic ecosystems. Microbial. Mol. Biol. Rev. 64 (2000), No. 1, 69-114.

[23] M. Begon et al. Population and transmission dynamics of cowpox in bank voles: testing fundamental assumptions. Ecol. 1 (1998), 82-86.

[24] M. Begon et al. Transmission dynamics of a zoonotic pathogen within and between wildlife host species. Proc R. Soc. Lond. B,266 (1999), 1939-1945.

[25] M.E. Hochberg. The potential role of pathogens in pest control. Nature, 337 (1989), 262-265.

[26] M. Inoue, H. Kamifukumoto. Scenarios leading to chaos in a forced Lotka Volterra model. Prog. Theor. Phys. 71 (1984), 930-937.

[27] O. Bergh, K.Y. Borsheim, G.Bratbak, M. Heldal. High abundance of viruses found in aquatic environments. Nature 340 (1989), 467-468.

[28] R.M. Anderson. Theoretical basis for the use of pathogens as biological control agents of pest species. Parasitology 84 (1982), 3-33.

[29] S. Busenberg, K.S. Kishore, P.Austin, G. Wake. The dynamics of a model of a planktonnutrient interaction. J. Math. Biol. 52 (1990), 677-696.

[30] S. Rinaldi, S. Murtori, Y. Kuznetsov. Multiple attractors catastrophe and chaos in seasonally perturbed predator-prey communities. Bull.Math.Biol. 55 (1993), 15-35.

[31] S. Ruan. Persistence and co-existence in zooplankton-phytoplankton-nutrient models with instantaneous nutrient recycling. J. Math. Biol. 31 (1993), 633-654.

[32] V.A. Pliss. Nonlocal problems of the theory of oscillations., Academic Press, New York, 1966. 